\title{
ACTIVIDAD FÍSICA ADAPTADA EN EL PROCESO DE REHABILITACIÓN DE PERSONAS CON DISCAPACIDAD: UNA PROPUESTA DESDE LA PERSPECTIVA SOCIAL
}

\section{ADAPTED PHYSICAL ACTIVITY IN THE REHABILITATION PROCESS FOR PEOPLE WITH DISABILITIES A PROPOSAL FROM THE SOCIAL PERSPECTIVE}

\author{
Fernando Muñoz Hinrichsen ${ }^{1}$ y Alan Martínez Aros ${ }^{2}$ \\ fernando.munoz h@umce.cl; alankine@gmail.com \\ 1 Universidad Metropolitana de Ciencias de la Educación, Santiago, Chile \\ Universidad Andrés Bello, Santiago, Chile
}

Envio original: 2021-05-18 Reenviado: 2021-08-13 Aceptado: 2021-12-03

Publicado: 2022-1-13

Doi: $\underline{\text { https://doi.org/10.15517/pensarmov.v20i1.47007 }}$

\begin{abstract}
RESUMEN
El proceso de rehabilitación es fundamental para las personas con discapacidad, y desde un marco ecológico bajo un enfoque social, es relevante pensar que la actividad física adaptada se convierte en una herramienta vital para el desarrollo integral bajo un enfoque de derechos humanos. El objetivo de este ensayo es dar relevancia a la actividad física adaptada, y denotar como se convierte en un espacio expresión social importante a la hora de desarrollar procesos de rehabilitación e inclusión social, junto con conocer cuáles son los alcances y recomendaciones a la hora de abordarlos. El desarrollo profesional es fundamental, evidenciado por procesos de investigación adecuados, y generar redes que permitan el trabajo comunitario para que el enfoque se base desde una perspectiva social. En conclusión, es fundamental entender la actividad física como un determinante social para la rehabilitación de personas con discapacidad, y así dar pie para desarrollar planes y programas que se enfoquen en los lineamientos internacionales de la rehabilitación basada en la comunidad con un modelo ecológico.
\end{abstract}

Palabras clave: discapacidad, rehabilitación, actividad física, calidad de vida 


\begin{abstract}
The rehabilitation process is a fundamental space for people with disabilities, and from an ecological framework under a social approach, it is relevant to think that adapted physical activity becomes a vital tool for comprehensive development under a human rights approach. The objective of this article is to give relevance to adapted physical activity, and denote how it becomes an important social expression space when developing rehabilitation and social inclusion processes, along with knowing what the scope and recommendations are when it comes to address them. Professional development is essential, evidenced by adequate research processes, and generating networks that allow community work so that the approach is based from a social perspective. In conclusion, it is essential to understand physical activity as a social determinant for the rehabilitation of people with disabilities, and thus give rise to the development of plans and programs that focus on international guidelines for community-based rehabilitation with an ecological model.
\end{abstract}

Keywords: disability, rehabilitation, physical activity, quality of life

\title{
INTRODUCCIÓN
}

El concepto actual de rehabilitación, propone un conjunto de intervenciones desarrolladas para optimizar el funcionamiento y reducir la discapacidad en individuos con condiciones de salud en interacción con su entorno (World Health Organization [WHO], $\underline{2010}$ ). Esto se relaciona con lo que se propone para el concepto de discapacidad, que plantea esta relación entre las características de la persona y el entorno en el que vive (WHO, 2011). Ambas concepciones se enmarcan en el modelo de la clasificación internacional de funcionamiento, discapacidad y salud (WHO, 2001), que se contextualiza en la teoría ecológica que relaciona de la misma manera a la persona, la tarea a realizar y el medio ambiente (Torrico Linares, et al., 2002). La nueva estrategia para la inclusión de personas con discapacidad (PCD de ahora en adelante) de la Organización de Naciones Unidas propone como clave 3 puntos que son el enfoque bidireccional, lo que significa que debe abordarse de manera integral, pero también específicamente de acuerdo a las necesidades, la intersectorialidad en la que los procesos inclusivos deben abordarse en conjunto con otros temas como la edad, el género y la geolocalización, ya que también son determinantes para las PCD, y finalmente la coordinación, fundamental para acelerar 
los procesos, aprender de las experiencias previas y lograr la inclusión como objetivo final (WHO, 2020). Lo anterior es muy relevante ya que está directamente vinculado a los objetivos del desarrollo sostenible, que son la educación, calidad, trabajo decente y crecimiento económico, la reducción de desigualdades, ciudades y comunidades sostenibles, y finalmente la alianza para lograr los objetivos (Comite Español de Representantes de Personas con Discapacidad, 2019).

La estrategia de "Rehabilitación Basada en la Comunidad" (RBC) se ha utilizado para resguardar los puntos previamente desarrollados, con el fin de dar mayor cobertura a la rehabilitación como derecho social, buscando incrementar la participación de las comunidades con sentido de pertenencia, fortalecer la colaboración multisectorial, incluir organización de personas con discapacidad y, lo que es más importante, ampliar y desarrollar programas basados en evidencia para la práctica (Tamayo, 2020).

Si lo llevamos a esta perspectiva y entendemos que la rehabilitación no es solo un proceso biomédico, sino orientado a la diversidad y la sociedad (Guajardo Cordoba, 2014), la actividad física adaptada toma mucha fuerza como lugar en esta integralidad. En primer lugar, porque tiene un enfoque también desde esta teoría ecológica planteada previamente bajo el modelo ecológico sistemático de actividad física adaptada (Hutzler, 2007), considerando la persona y sus propias características, a la tarea desde la actividad o ejercicio físico, y finalmente desde el entorno, en el que se ejecuta desde su particularidad. En segundo lugar, dado que, desde su conceptualización, la Federación Internacional de Actividad Física Adaptada propone que la actividad física adaptada (AFA) es una rama profesional de la kinesiología / educación física / ciencias del deporte y del movimiento humano, la cual está dirigida a personas que requieren adaptación para participar en el contexto de la actividad física (Sherrill, 2008). Esto se puede entender a partir de un modelo que pretende visualizar que es un beneficio a nivel físico, emocional, social y comunitario, por lo que sería de gran aporte al proceso de rehabilitación. Sin duda, también podría consignarse como parte de los determinantes sociales, ya que, si bien la discapacidad se considera un aspecto puramente de salud, desde el modelo ecológico podemos entenderla como un determinante social estructural (Tamayo, 2018) el cual podría sumarse como parte de los procesos de inclusión/exclusión de la estratificación social e involucrarse en aspectos como educación, etnia, género, y así generar políticas de exposición y vulnerabilidad. La actividad física cobra gran relevancia debido a que es considerada como una herramienta que permite la inclusión social por sus características relacionadas con la mejora de la salud mental, el bienestar y las 
capacidades psicológicas mediante el fortalecimiento de la seguridad corporal, la autoestima y la confianza en uno mismo, reducir el estrés, la ansiedad y la depresión, aumentar la función cognitiva y desarrollar una amplia gama de habilidades y cualidades, como la cooperación, la comunicación, el liderazgo, la disciplina, el trabajo en equipo, que contribuyen al éxito en el juego y el aprendizaje y en otros aspectos de la vida (Organización de las Naciones Unidas para la Educación, la Ciencia y la Cultura, 2015).

El objetivo de este ensayo es mostrar cómo la actividad física adaptada se convierte en una herramienta importante a la hora de desarrollar procesos de rehabilitación con un enfoque inclusivo para las personas con discapacidad.

\section{UNA MIRADA DESDE EL DESARROLLO HISTÓRICO}

En los comienzos de la AFA, esta se propone como parte de iniciativas relacionadas a la rehabilitación, en particular de personas con secuelas físicas de la segunda guerra mundial y que tuvieron afectados el sistema nervioso y la medula espinal. Este comienzo fue entre los años 1940 y 1950 en Inglaterra gracias al trabajo del Doctor Ludwig Guttmann. Es muy relevante recordar este acontecimiento, ya que de esta forma se logra comprender de modo efectivo como pasa a ser un proceso importante para las PCD en el contexto de salud, el cual nace y evoluciona con el fin de garantizar los procesos de inclusión y participación social (Gil, et al., 2013). La inclusión de programas de AFA en centros de salud es una necesidad que se requiere en la actualidad, esto en conjunto con los requerimientos de reconocer a los profesionales de la actividad física y el deporte los cuales deben estar incluidos en el equipo de trabajo de estos centros como ya ocurre en países de Europa (Ruiz, 2007; Gil, et al., 2013).

El desarrollo deportivo ha mostrado un crecimiento sostenido en centros de rehabilitación, por ejemplo en el Hospital Nacional de Parapléjicos de Toledo o el Instituto Guttman de Barcelona, ambos en España y en donde existen clubes que desarrollan diversos tipos de actividades y deportes. A estos se suman los centros de referencia en discapacidad que ya están comenzando a introducir la actividad física deportiva en sus servicios tales como el Centro Estatal de Atención al Daño Cerebral de Madrid, el Centro de Rehabilitación de San Andrés del Rabanedo en la ciudad de León (Garcia-Hernández, 2013). En Chile el Instituto Nacional de Rehabilitación Pedro Aguirre Cerda, los Institutos Teletón y los Centros Comunitarios de Rehabilitación también han sido instancias que han buscado implementar estos programas, lo que da lugar para entender la relevancia de estos en el modelo de abordaje para la habilitación y rehabilitación. Es muy importante la 
relación y el compromiso entre el área de la salud y los niveles de iniciación en el ámbito y área del deporte, existen clubes deportivos por ejemplo que poseen una orientación inclusiva desde una perspectiva de rehabilitación, y que están dirigidos a la gestión del proceso deportivo de las PCD como por ejemplo el Club Deportivo elemental de Lesión Cerebral Adquirida de Madrid (Pérez-Tejero, 2009), que muestran que esta relación se debe favorecer entre estas dos áreas. Nuevamente Chile ha tomado esta iniciativa y tanto el Instituto Nacional de Rehabilitación como los centros de Teletón y los Comunitarios de Rehabilitación han adoptado la misma estrategia y se han transformado en parte activa de organizaciones como el Comité Paralímpico. La actividad físico-deportiva ha jugado un papel de gran importancia para las personas con discapacidad, y se alinea con los beneficios de la actividad física para la salud de la población en general, con la cual estamos totalmente de acuerdo (Vallbona, 2003).

\section{BENEFICIOS DE LA ACTIVIDAD FÍSICA ADAPTADA EN EL CONTEXTO DE LA REHABILITACIÓN}

La actividad física en el proceso terapéutico, optimiza los modelos de rehabilitación, reeducación o rehabilitación de PCD (Segura, et al., 2013). Definitivamente, la rehabilitación busca fortalecer las capacidades abolidas para las personas, con el objetivo de que puedan desenvolverse en su contexto. Según Segura et al. (2013) el desarrollo de la AFA se encuentra dirigido principalmente a:

- La optimización y recuperación de las capacidades físicas, sensoriales y/o mentales afectadas.

- Aportar en los procesos personales vinculados a la autoestima, afianzando la confianza en sus habilidades y/o competencias.

- Fortalecer la salud desde la amplitud del concepto con una perspectiva holística.

Desde esta perspectiva se transforma en una herramienta de mucha relevancia e importancia en el proceso de la rehabilitación relacionando beneficios físicos funcionales, psicológicos, sociales y generales que contribuyen a un bienestar multicomponente que a su vez se relaciona como un sistema (ver Figura 1). 


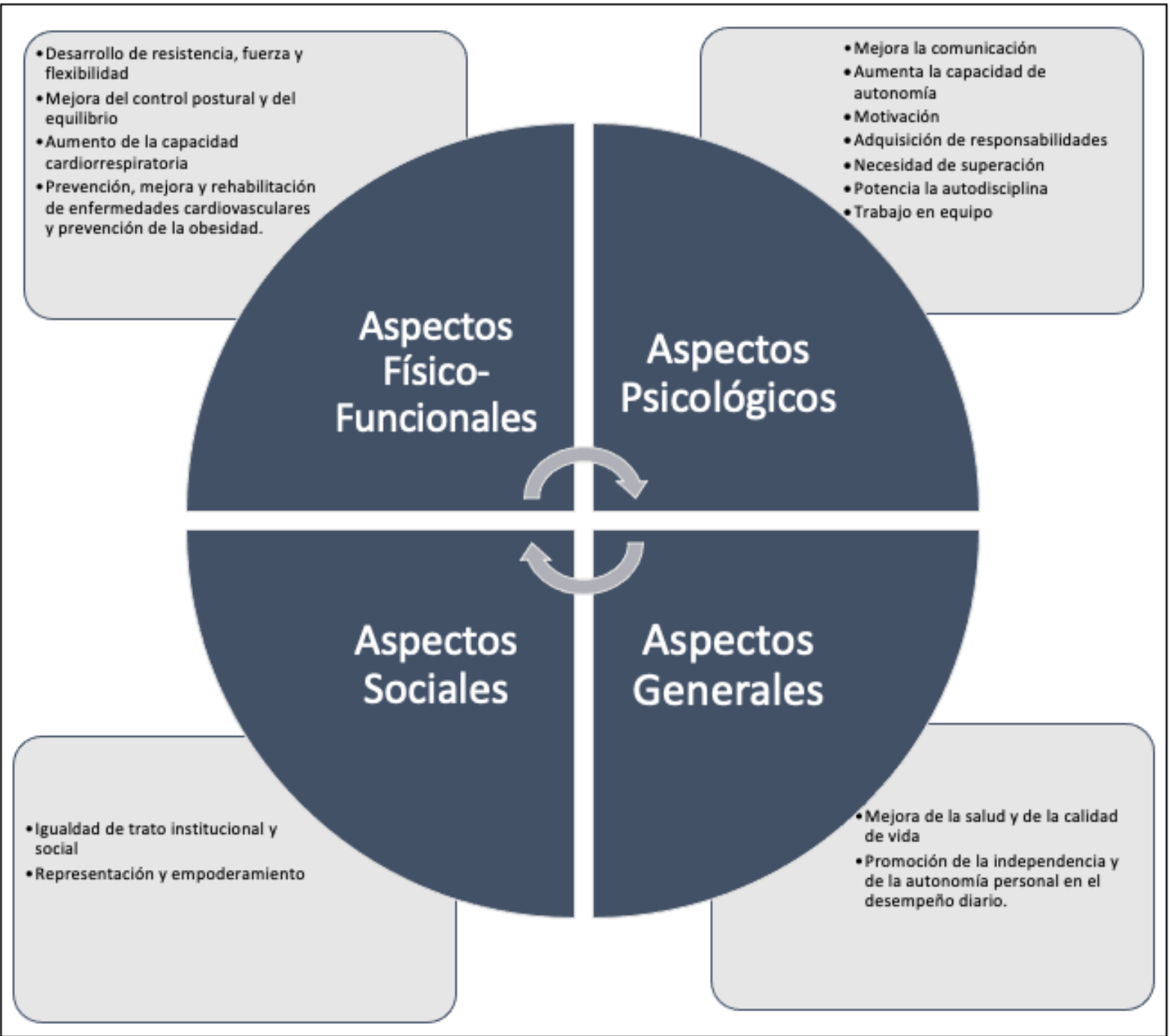

Figura 1. Beneficios de la práctica de actividad física adaptada para personas con discapacidad. Fuente: adaptado de Monforte et al. (2019).

Durante la Conferencia Internacional de Deportes Adaptados (Pérez-Tejero, 2009) se establece claramente que el deporte adaptado no tiene por qué llevar adjetivos: es deporte. El deportista paralímpico tiene derecho a ser reconocido en igualdad de trato deportivo, institucional y social. 


\section{DESARROLLO DE LA ACTIVIDAD FísICA ADAPTADA EN EL CONTEXTO DE LA REHABILITACIÓN}

Al generar una propuesta de desarrollo, se deben considerar algunos aspectos esenciales para que su implementación sea lo más adecuada posible (Ver figura 2).

1. A partir de una macro propuesta pensada en el modelo ecológico (Torrico Linares, et al., 2002) se debe abordar una macroestructura que en este caso particular surge de los determinantes sociales de la salud en primera instancia (Tamayo, 2018), tomando en consideración el modelo desde lo estructural en la que la discapacidad se convierte en un factor fundamental. A su vez, se relaciona con el contexto social desde la perspectiva laboral y educativa, como aspectos fundamentales para la protección de los derechos de las personas con discapacidad, a partir de la propuesta de la Convención Internacional de la ONU (World Health Organization, 2006) en el artículo 24 y 27.

2. Teniendo clara la macroestructura a la que se asigna la propuesta de desarrollo, se proponen los espacios en los que se puede aplicar el modelo, desde instituciones de rehabilitación regulares con un enfoque técnico en procesos y fases agudas, hasta un avance en los centros de desarrollo de la comunidad vinculados a la práctica de la actividad física y el deporte como espacios sociales inclusivos y diseñados en un acercamiento a la diversidad de expresiones. A su vez, cómo esto se relaciona con los campos más específicos.

3. A partir de estos campos específicos, y de las acciones que se puedan desarrollar, hay que tomar en consideración una evolución en complejidad y observarla desde la teoría de la formación (Wing, 2012) en conjunto con los procesos de rehabilitación y sus principales actores en la actividad física adaptada planteados por la Federación Internacional de la Actividad Física Adaptada (2021). La fisioterapia en centros de rehabilitación pasa a tomar un rol fundamental, dirigida a personas con discapacidad en etapas agudas de rehabilitación, y realizada por equipos de profesionales de la salud especializados en el área de la AFA. Al mismo tiempo en un puesto de trabajo en conexión y comunicación con los centros educativos o laborales para un trabajo conjunto. La actividad física está dirigida principalmente a la recuperación o adquisición de componentes del entrenamiento físico (Wing, 2012) y patrones de movimiento que se relacionan con el movimiento 
óptimo, pero considerando la diversidad y adaptaciones al mismo, siempre con una orientación hacia las capacidades y no a las limitaciones.

4. Ejercicio físico: Esto en una transición en las modalidades de atención hacia procesos ambulatorios en fase subaguda desde su condición, conducida a la actividad por equipos profesionales que pueden desarrollarse en los espacios de salud, pero con conexión directa a los equipos de trabajo en los distintos espacios sociales, y que a su vez ya está apalancado con procesos de adaptación para el desempeño en sus centros educativos o laborales. En esta etapa el trabajo ya se vuelve complejo debido a que requiere contextualización con el entorno, por lo que la comunicación es clave entre equipos. Desde el ejercicio físico, el trabajo está basado en los principios del entrenamiento la especificidad, la progresión de la carga y el control de esta (Wing, 2012). Para generar las adaptaciones y adecuaciones, el "Modelo sistemático Ecológico en Actividad Física Adaptada" (SEMA) sería de gran aporte, porque permite hacer una relación entre las necesidades de las personas, en el cómo aplicar los ajustes al entorno y en la tarea, lo que da una visión contextualizada para la práctica de AFA (Hutzler, 2007). Este sistema también podría aplicarse en la etapa anterior relacionada con la fisioterapia ya que los mismos principios podrían ser considerados desde un enfoque y marco ecológico.

5. Actividad Física y Deporte Adaptado/Inclusivo: en esta etapa y estrategia, las actividades pueden ser llevadas al entorno comunitario, teniendo en cuenta la posibilidad de que las personas puedan acceder a actividades diseñadas para ellos, como las que van de la mano con el deporte adaptado (Leardy, 2018), o aquellas que se consideren en el marco de la inclusión como el derecho a participar en actividades de libre elección bajo el concepto de accesibilidad en todos los sentidos (estructural, social y desde la comunicación). Es en este espacio se debe generar una conexión y compromiso con los profesionales que trabajan en los distintos campos de la AFA, por lo que la comunicación y el trabajo entre las distintas disciplinas es fundamental. Debe garantizarse una oferta oportuna para cubrir las necesidades de la PCD (World Health Organization, 2006), y aquí se debe establecer el vínculo con las instituciones formales del deporte adaptado (como los Comités Paralímpicos, Olimpiadas Especiales o Olimpiadas para Sordos, para así acomodarse a la práctica deportiva regulada y bajo la estructura internacional en relación a ampliar la oferta, que a su vez deben 
generar instancias de libre práctica, en las que el hábito, y por supuesto los procesos de adherencia a la práctica son fundamentales de la AFA, con especial énfasis en aspectos y beneficios de salud, bienestar y calidad de vida.

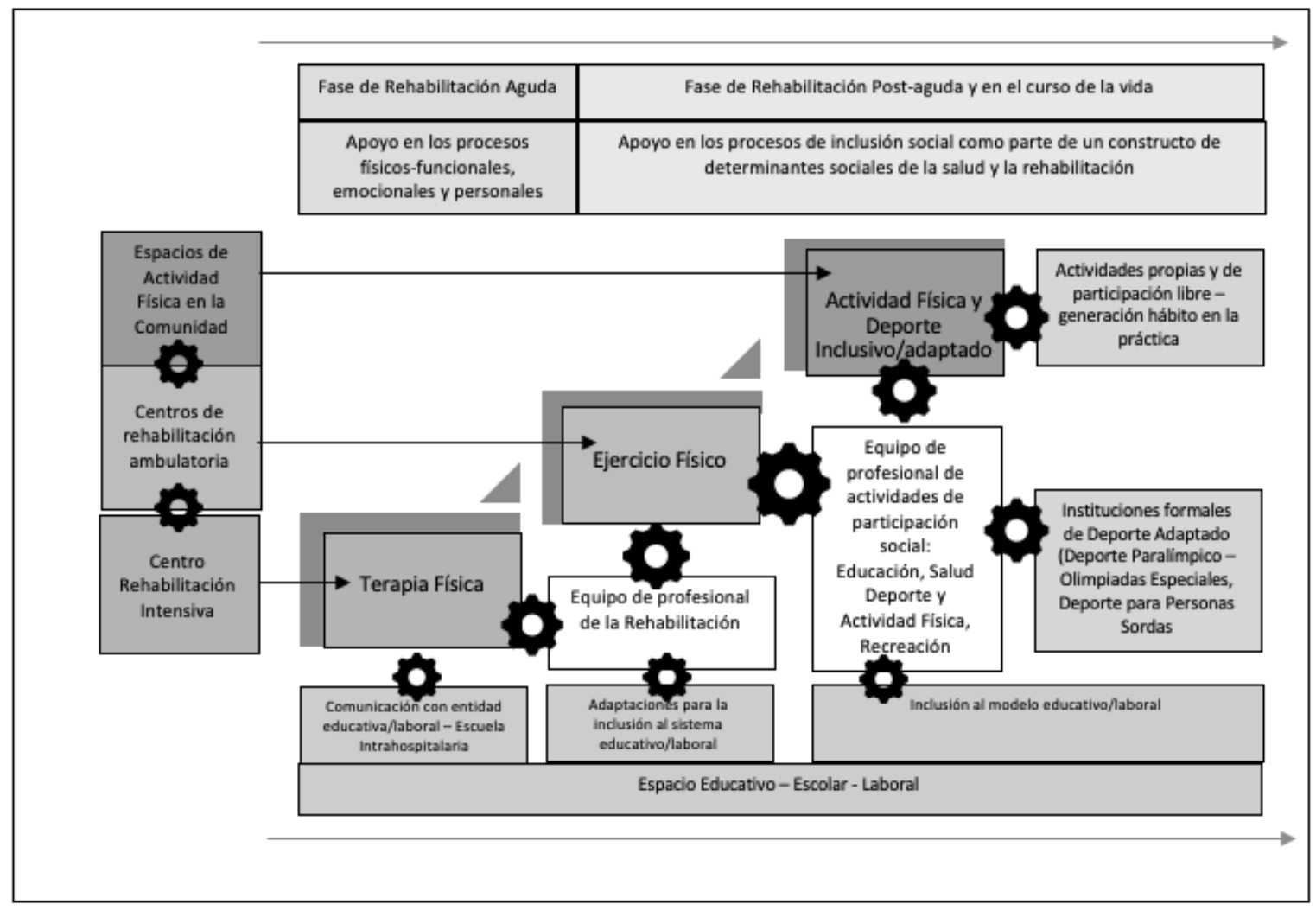

Figura 2. Propuesta de modelo de desarrollo para AFA en el contexto de la rehabilitación. Fuente: adaptación de la propuesta del modelo AFA de promoción de la salud en rehabilitación (Rimmer, 1999; Schüle y Huber, 2004).

\section{CONSIDERACIONES PARA LA ACTIVIDAD FÍSICA ADAPTADA EN EL CONTEXTO DE LA REHABILITACIÓN:}

- Se vuelve una necesidad fundamental que existan procesos de formación en el área y desarrollo de experiencias como herramienta de mejora. Esto ya ha sido planteado por varios espacios vinculados a la temática (Muñoz-Hinrichsen, et al., 2020) que han ido entendiendo a partir de las experiencias, el desarrollo de proyectos y las opiniones de sus actores, que tener un espacio para adquirir conocimiento es un pilar para este campo. 
- Evidenciar desde el marco científico los procesos de intervención. Esto da la posibilidad de conocer cuáles son las necesidades reales con una base científica y sólida para poder dar solución al desarrollo en el campo de la rehabilitación. Existe un sin número de espacios de desarrollo, desde la perspectiva de la estructura y la función corporal, desde la investigación en la actividad y la participación social, y a esto se suman los factores personales y del entorno (Pérez-Tejero, 2012).

- Considerar propuestas de programas desde el marco social con enfoque en lo que requiere la comunidad. Esto se basa principalmente en las propuestas de rehabilitación comunitaria, que se convierte en la principal estrategia para abordar una intervención en un modelo ecológico, bajo pautas internacionales. A esto se suma la generación de redes adecuadas y conciencia sobre la importancia de la gestión y los recursos (Guajardo Cordoba, 2014).

\section{CONCLUSIÓN}

Es trascendental entender y analizar la actividad física como un determinante social desde la amplitud de su desarrollo e implementación para la rehabilitación de PCD, considerando todos sus beneficios y aportes en los aspectos físicos, emocionales y psicosociales, y a su vez siendo un espacio de expresión social relevante que da la posibilidad de el empoderamiento, la visualización, socialización e inclusión efectiva. Esto permitiría dar pie para desarrollar planes y programas que se enfoquen en los lineamientos internacionales de la rehabilitación, basada en la comunidad con un modelo ecológico, que tiene directa relación con la clasificación internacional del funcionamiento y el entender la discapacidad como una relación entre las características de la persona en conjunto con el entorno que la rodea, desde las personas facilitadoras y barreras que la comunidad presenta para la participación efectiva.

\section{REFERENCIAS}

Comité Español de Representantes de Personas con Discapacidad. (2019). Objetivos del desarrollo sostenible y promoción de los derechos de las personas con discapacidad. Grupo Editorial Cinca.

Federación Internacional de Actividad Física Adaptada. (2021). Web Site IFAPA. https://ifapa.net/what-is-apa/ 
García-Hernández J., Mediavilla-Saldaña L., Pérez-Rodríguez M., Pérez-Tejero, J. y González-Alted, C. (2013). Análisis del efecto de las actividades físicas grupales en pacientes con daño cerebral adquirido en fase subaguda. Revista Neurología, 57(2), 64-70. https://doi.org/10.33588/rn.5702.2012634

Gil, J. L., García-Unanue, J., Felipe, J. L., Gallardo, L. y Burillo, P. (2013). Demandas de los

profesores de educación física para una enseñanza de calidad respecto a las instalaciones deportivas escolares. Journal of Sports Economics \& Management, $3(1)$,

64-77.

\section{http://sportsem.uv.es/j sports and em/index.php/JSEM/article/view/23/23}

Guajardo Cordoba, A. (2014). Rehabilitación de Base Comunitaria Diálogos, reflexiones y prácticas en Chile. Servicio Nacional de la Discapacidad.

Hutzler, Y. (2007). A Systematic Ecological Model for Adapting Physical Activities: Theoretical Foundations and Practical Examples. Adapted Physical Activity Quarterly, (24), 287-304. https://doi.org/10.1123/apaq.24.4.287

Leardy, L. (2018). Cuestiones terminológicas referidas al deporte de personas con discapacidad. En: Libro Blanco del Deporte para Personas con Discapacidad (pp. 21-25). Grupo Editorial Cinca.

Monforte, J., Úbeda-Colomer, J., Smith, B. y Foster, C. (2019). Infografía sobre actividad física para personas adultas con discapacidad. Revista Española de Discapacidad, 7(1), 257-265. https://www.cedd.net/redis/index.php/redis/article/view/532

Muñoz-Hinrichsen, F., Bossay. C., Henriquez, M., Martinez, A. y Castelli, L.F. (2020). Deporte paralímpico en Chile, una aproximación a la realidad nacional en el año 2019. Revista Iberoamericana de Ciencias de la Actividad Física y el Deporte, 9(3), 91-101. https://doi.org/10.24310/riccafd.2020.v9i3.8575

Organización de Naciones Unidas para la Educación, la Ciencia y la Cultura [UNESCO]. (2015). International Charter of Physical Education, Physical Activity and Sport. https://unesdoc.unesco.org/ark:/48223/pf0000235409

Pérez-Tejero, J. (2009). La Investigación en Actividades Físicas y Deportes Adaptados: un camino aun por recorrer. Revista Internacional de Ciencias del Deporte, V(16), 1-3. https://www.redalyc.org/pdf/710/71014352001.pdf

Pérez-Tejero, J., Reina, R. y Sanz, D. (2012). La Actividad Física Adaptada para personas con discapacidad en España: perspectivas científicas y de aplicación actual. 
Cultura, Ciencia y $\quad$ Deporte, $\quad 7(8), \quad$ 213-224. http://www.redalyc.org/articulo.oa? $\mathrm{id}=163024688008$

Rimmer, J.H. (1999). Health promotion for people with disabilities: the emerging paradigm shift from disability prevention to prevention of secondary conditions. Physical Therapy, 79(5), 495-502. https://pubmed.ncbi.nlm.nih.gov/10331753/

Ruiz, P. (2007). Estado de la cuestión en la formación en AFA en España y Europa. En: II Conferencia Internacional sobre Deporte Adaptado. Instituto Andaluz del Deporte.

Schüle, K. y Huber, G. (2004). Essentials of sport therapy [Grundlagen der Sporttherapie] ( $2^{\text {nd }}$ ed.). Elsevier; Urban, \& Fischer.

Segura, J., Martínez-Ferrer, J-O. y Barnet, S. (2013). Creencias sobre la inclusión social y el deporte adaptado de deportistas, técnicos y gestores de federaciones deportivas de deportes para personas con discapacidad. Revista Iberoamericana de Psicología del Ejercicio y el Deporte, 8(1), 120-144. https://www.redalyc.org/pdf/3111/311127595008.pdf

Sherrill, C. y Hutzler, Y. (2008). Adapted physical activity sciences. In J. Borms (Ed.), Directory of sport science $\left(5^{\text {th }}\right.$ ed). ICSSPE/CIEPSS.

Tamayo, M. (2018). Determinantes sociales de la salud y discapacidad: actualizando el modelo de determinación. Gaceta Sanit, 32(1), 96-100. https://doi.org/10.1016/j.gaceta.2016.12.004

Tamayo Rozas, M. (2020). Kinesiología y discapacidad: perspectivas para una práctica basada en derechos. Universidad de Chile.

Torrico Linares, E., Santín, C., Villas, M., Álvarez-Dardet, S. y López, M.J. (2002). El modelo ecológico de Bronfrenbrenner como marco teórico de la Psicooncología. Anales de Psicología, 18(1), 45-59. https://www.redalyc.org/pdf/167/16718103.pdf

Vallbona, C. (2003). La actividad física como elemento de salud para personas discapacitadas. Instituto Andaluz del Deporte.

Wing, C. (2012). ACSM/NCHPAD Resources for the Inclusive Fitness Trainer. American College of Sports Medicine.

World Health Organization [WHO]. (2001). International classification of functioning, disability and health. Instituto de Mayores y Servicios Sociales.

World Healt Organization [WHO]. (2006). Convention on the Rights of Persons with Disabilities. Ney York, United Nation.

World Health Organization [WHO]. (2010). Community-based rehabilitation: $\mathrm{cBr}$ guidelines. Inís communication. 
World Health Organization [WHO]. (2011). World Report on Disability. Library Cataloguingin-Publication Data.

World Health Organization [WHO]. (2020). United Nation Disability Inclusion Strategy. Ginebra, United Nation. 